\title{
RRHP: a tag-based approach for 5-hydroxymethylcytosine mapping at single-site resolution
}

\author{
Adam Petterson ${ }^{1,2+}$, Tzu Hung Chung ${ }^{1 \dagger}$, Darany Tan ${ }^{1}$, Xueguang Sun ${ }^{1 *}$ and Xi-Yu Jia ${ }^{1}$
}

\begin{abstract}
Current methods for genomic mapping of 5-hydroxymethylcytosine ( $5 \mathrm{hmC}$ ) have been limited by either costly sequencing depth, high DNA input, or lack of single-base resolution. We present an approach called Reduced Representation 5-Hydroxymethylcytosine Profiling (RRHP) to map 5hmC sites at single-base resolution by exploiting the use of beta-glucosyltransferase to inhibit enzymatic digestion at the junction where adapters are ligated to a genomic library. Therefore, only library fragments presenting glucosylated $5 \mathrm{hmC}$ residues at the junction are sequenced. RRHP can detect sites with low 5hmC abundance, and when combined with RRBS data, 5-methylcytosine and 5-hydroxymethylcytosine can be compared at a specific site.
\end{abstract}

\section{Background}

Since 2009, one of the most rapidly developing subdisciplines in molecular genetics has proven to be the identification and characterization of 5-hydroxymethylcytosine $(5 \mathrm{hmC})$. Like its close relative, 5-methylcytosine $(5 \mathrm{mC})$, $5 \mathrm{hmC}$ is one of the covalent modifications observed in prokaryotic and eukaryotic genomes [1,2], which constitute an important class of epigenetic modifications. At present, the precise role of $5 \mathrm{hmC}$ in the genomic context is under close study from a myriad of angles. One paradigm implicates $5 \mathrm{hmC}$ in the oxidative demethylation of cytosine [3], which has been bolstered by subsequent characterizations of 5 formylcytosine $(5 \mathrm{fC})$ and 5-carboxylcytosine $(5 \mathrm{caC})$ in the genome [4]. Aside from its mechanistic characterization, $5 \mathrm{hmC}$ localization and tissue distributions have also been extensively studied, resulting in clear demonstration of elevated abundance in tissues within the central nervous system (CNS) [5]. Pathologically, a profound depletion of $5 \mathrm{hmC}$ is observed across several malignant carcinomas [6].

As a result of the increased study of this modification, more sensitive tools are required for detection, quantitation, and ultimate mapping of the marker across the genome. While methods such as LC-MS/MS-MRM are

\footnotetext{
* Correspondence: xsun@zymoresearch.com

${ }^{\dagger}$ Equal contributors

'Zymo Research Corporation, 17062 Murphy Ave., Irvine, CA 92614, USA

Full list of author information is available at the end of the article
}

useful for sensitive detection and quantitation of $5 \mathrm{hmC}$ and other modified nucleosides, most genetic applications require the ability to pin down the mark to a tight region, locus, or specific junction within a locus. Several technologies have become available that utilize old methodologies, such as immunoprecipitation or qPCR, as well as new methodologies, including chemical labeling, singlemolecule kinetic monitoring, nanopore conductivity, and more. A recent review highlights the advantages and pitfalls of these techniques [7].

For many applications, genome-wide approaches, including hMeDIP and bio-orthogonal labeling with glucosylation, provide robust enrichment pools for Sanger sequencing as well as massively parallel (next-generation) sequencing. Despite good coverage of the genome and high specificities, these methods are often limited by input requirements which typically are in the neighborhoods of several micrograms. These amounts of DNA are often not feasible for investigation of precious samples such as stem cells or selectively isolated cellular subpopulations (that is, diverse neuronal cells from a whole brain sample). Importantly, these enrichment-based methodologies, even in highly optimized protocols, lack single-base resolution, and identified hydroxymethylated sites will fall within the range of several hundred to several thousand bases. Depending on how well a particular region is annotated, such resolution is often insufficient to describe activity 
in transcriptionally relevant sites with confidence. Findings from such studies require subsequent validations with locus-specific assays, such as glucMS-qPCR, to enhance the $5 \mathrm{hmC}$ positions.

Recently, two approaches which enable quantitative, single-base resolution mapping of $5 \mathrm{hmC}$ have been reported. Oxidative bisulfite sequencing (oxBS-Seq) [8] takes advantage of selective chemical oxidation via organometallic catalysis to yield $5 \mathrm{fC}$ from $5 \mathrm{hmC}$, which is then susceptible to traditional bisulfite conversion and results in a different sequencing signal from the $5 \mathrm{mC}$ sibling. The $5 \mathrm{hmC}$ level is inferred by comparing the methylation values between the modified and traditional bisulfite sequencing. Although this process allows interrogating of $5 \mathrm{hmC}$ at single-base resolution, the oxidation step leads to significant DNA degradation (approximately $0.5 \%$ of original DNA fragments are retained through the process, according to the authors), which again restricts its application to very rare samples. In addition to this approach, great strides have been reported with the Tet-assisted Bisulfite Sequencing (TAB-Seq) [9] approach. In this methodology, 5hmC positions are initially protected by glucosylation and then treated with the Tet enzyme to selectively oxidize naked $5 \mathrm{mC}$ positions to $5 \mathrm{hmC}$ and then $5 \mathrm{fC}$ and $5 \mathrm{caC}$. These $5 \mathrm{fC}$ or $5 \mathrm{caC}$ positions are susceptible to bisulfite conversion and deamination, so the only remaining cytosine positions are those originating from $5 \mathrm{hmC}$. While the method avoids harsh organometallic treatment for oxidation, it extensively depends upon the Tet enzyme, which is known to present low efficiency (the authors suggested an efficiency of $\geq 90 \%$, which can render at least $10 \%$ of methylated residues unconverted) [9]. Unconverted positions would, therefore, be falsely identified as $5 \mathrm{hmC}$ sites and contribute to a higher background signal for the assay.

As an alternative to these methods, we present a novel approach, known as reduced representation 5hydroxymethylcytosine profiling (RRHP), that avoids harsh chemical conversion processes and affords sequencelevel resolution of $5 \mathrm{hmC}$ positions. The method features a rapid workflow ( $<24$ total $h$ ), allows for starting inputs as low as $100 \mathrm{ng}$, and offers strand-specific information about $5 \mathrm{hmC}$ distribution. The absence of chemical conversions also allows for sequencing of native DNA sequences, which enhances sequencing quality and resulting mapping ratios. Most importantly, the method proves to be a highly reproducible, positive display method, allowing for higher confidence when interrogating positions with low $5 \mathrm{hmC}$ content. When combined with existing reduced representation bisulfite sequencing (RRBS) data for the same sample, RRHP allows for both high resolution and accurate quantitation of $5 \mathrm{mC}$ and $5 \mathrm{hmC}$ positions across the genome simultaneously.

\section{Results}

\section{Preparatory scheme and initial evaluation}

RRHP is dependent upon the availability of restriction endonucleases that exhibit insensitivity to methylation or hydroxymethylation within their cut sites while maintaining sensitivity to glucosyl modifications. In the simplest embodiment of the method, we digested human cerebellum DNA with MspI (which cleaves the $\mathrm{C}^{\vee} \mathrm{CG}^{\wedge} \mathrm{G}$ pattern, regardless of the $5 \mathrm{mC}$ or $5 \mathrm{hmC}$ status) and ligated the resulting fragments to modified adapters compatible with the Illumina TruSeq P5/P7 series. The adapters were designed such that the MspI site was reconstituted at the junction of the P5 adapter and DNA fragment but not at the junction of the P7 adapter and DNA fragment. $5 \mathrm{hmC}$ within the ligated library fragments were glucosylated with $\beta$-glucosyltransferase $(\beta$ GT). Fragments with a glycosylated $5 \mathrm{hmC}$ at the junction were resistant to a second MspI digestion while fragments with an unmodified $\mathrm{C}$ or a $5 \mathrm{mC}$ were cleaved, therefore, removing the P5 adapter and preventing the library fragments from being amplified. Following size selection and limited amplification, the libraries were sequenced using the standard Illumina TruSeq workflow without any modifications to the standard configurations or reagents (Figure 1). Each sequencing read with a CCGG tag at the beginning of the read represented one 5 hmC site.

To evaluate the method, we prepared six libraries (Figure 2a). Two negative control libraries (lane 1: without DNA input, lane 2: without the glucosylation step) demonstrated no observable product during the final amplification. Two duplicate libraries with $500 \mathrm{ng}$ DNA input (lane 4 and 5) gave product within the expected range, as did the library from $100 \mathrm{ng}$ DNA input (lane 6). We also prepared a library (lane 3) where the final cleavage was performed with HpaII, a methylation-sensitive isoschizomer, instead of MspI. This alternative digestion scheme allowed for the identification of any form of methylation at the adapter-fragment junction. A RRBS library [10] was also generated from the same brain sample for parallel comparison.

\section{Sequencing analysis}

We obtained 23 million reads for the RRHP library prepared from $500 \mathrm{ng}$ DNA input of which 95\% mapped to the reference genome and $95 \%$ of the mapped reads demonstrated a CCGG tag at the start of the read. In total, 1.74 million unique MspI sites were profiled, accounting for $94.3 \%$ of detectable MspI sites within fragments selected from 40 to 430 bp from an in silico digestion (Table 1). We observed high concordance in fragment distribution by overlaying experimental data with the simulation (Figure 2b). A large number of $5 \mathrm{hmC}$ sites (44\%) profiled were covered by less than five CCGG-tagged 


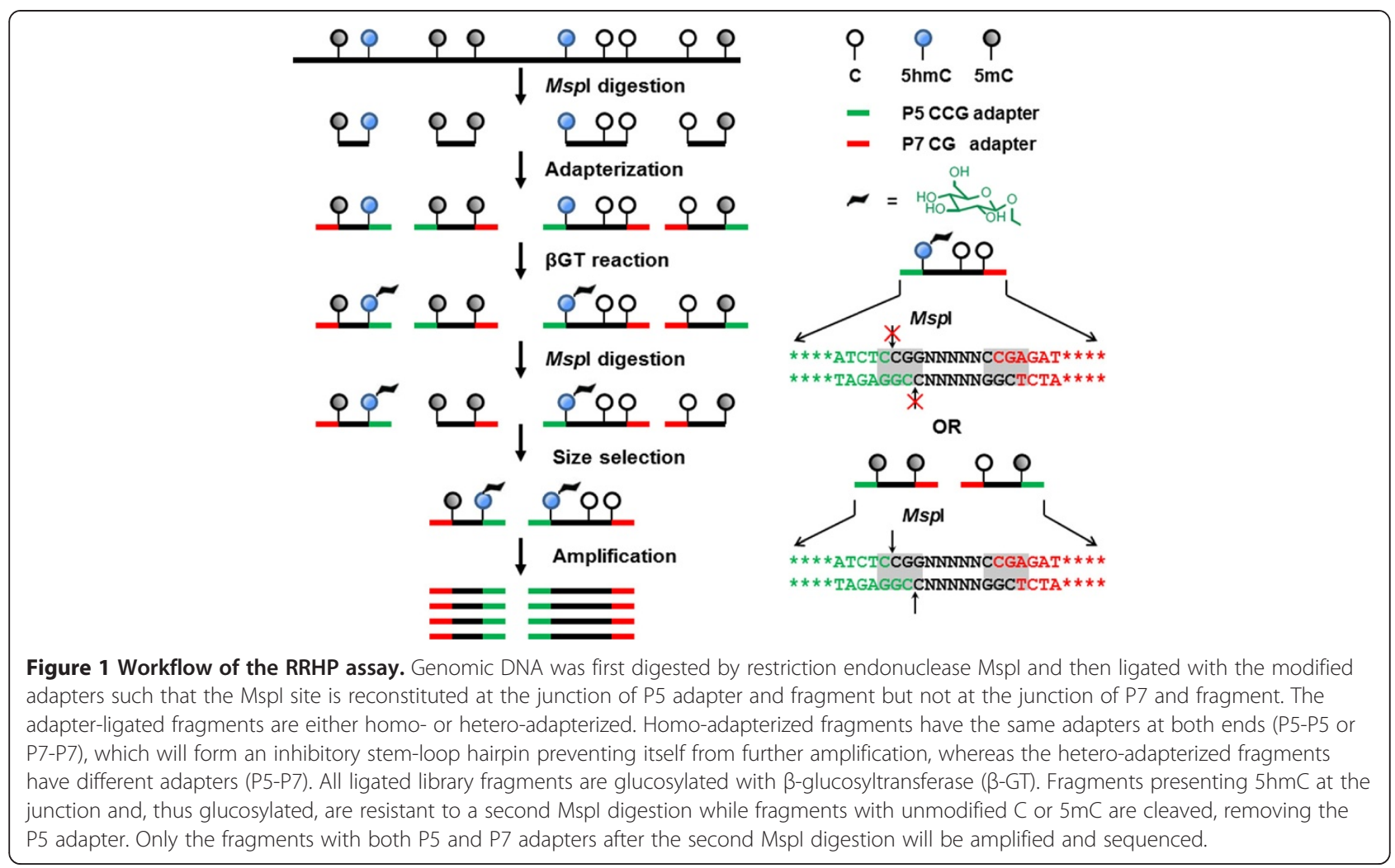

reads (Figure 2c and Additional file 1); since read number is proportional to $5 \mathrm{hmC}$ display, this indicated detection of $5 \mathrm{hmC}$ sites with relatively low abundance. We compared the HpaII-digested and MspI-digested libraries and observed that $85.6 \%$ of $5 \mathrm{mC}$ sites overlapped with $5 \mathrm{hmC}$ sites. Our results from RRHP agreed with observations from a TAB-sequencing study and supported the notion that $5 \mathrm{hmC}$ is ubiquitous in the genome but with a lower abundance than the $5 \mathrm{mC}$ mark [9]. Pairwise comparison between MspI libraries prepared with different DNA inputs (RRHP-MspI-1 and RRHP-MspI-3) showed a high concordance (Pearson's coefficient $=0.92$ ), and nearly 1.46 million $5 \mathrm{hmC}$ sites (83.3\%) overlapped between the two libraries (Figure 3a and 3c). To determine the effects of read depth on detection sensitivity, we sequenced one of the duplicated libraries (RRHP-MspI-2) with 50\% less depth and obtained roughly 10 million reads with similar mappability (94.9\%). Correlation between the replicates was lower than the samples with a higher read depth, but the Pearson's coefficient was still 0.86 and 1.32 million sites $(75 \%$ of the total profiled sites) overlapped in both libraries (Figure 3b and 3d). Further analysis showed that the non-overlapped sites between the two libraries had lower read counts compared with the overlapped sites (Figure 3e and 3f), and about 95\% of these sites had a read count below three. This indicated that higher sequencing depth is needed to enhance the reproducibility and to confirm the presence of $5 \mathrm{hmC}$ with low abundance.
By comparing the data from RRHP with RRBS, we were also able to estimate the minimal read counts needed to reach $<5 \%$ error rate in $5 \mathrm{hmC}$ detection. Since both RRHP and RRBS employ MspI for fragmentation during library preparation, we were able to simultaneously compare $5 \mathrm{hmC}$ with $5 \mathrm{mC}$ for certain sites. In principle, any CpG site with zero methylation in RRBS should not have any reads in RRHP. However, if there is a RRHP read detected in those sites, it could have resulted from a false calling. Based on that, we calculated the error rate for RRHP sites with various read counts by selecting CpG sites from RRBS with greater than $50 \times$ read coverage and zero methylation and cross-checking them with MspI cutting sites (CCGG) which were considered as potential detectable RRHP sites. In total, 1,635 CpG sites passed the criteria and this number was denoted as N. Any CpG site with i reads $(i \geq 1)$ from the RRHP assay was considered as a false hydroxymethylated site. The error rate was calculated as Error rate $\left(E_{i}\right)=$ Number of $\mathrm{N}_{\mathrm{i}} /$ Number of $\mathrm{N}$. As shown in Table 2, the false calling rate was less than $5 \%$ when read counts reached four or greater. If we exclude $5 \mathrm{hmC}$ sites with a low read coverage $(<5)$ to remove the noise introduced by spurious reads, interestingly, the percentage of overlapping sites did not change significantly between the technical replicates with the same sequencing depth (Additional file 2A). However, the correlation metrics increased constantly with the higher read cutoff (Additional file 2B). Due to the positive display nature 


\begin{tabular}{|cccccccc|}
\hline Lane & $\mathrm{L}$ & 1 & 2 & 3 & 4 & 5 & 6 \\
DNA $(\mu \mathrm{g})$ & & 0 & 0.5 & 0.5 & 0.5 & 0.5 & 0.1 \\
$\beta G T$ & & + & - & - & + & + & + \\
Mspl/Hpall & & $\mathrm{M}$ & $\mathrm{M}$ & $\mathrm{H}$ & $\mathrm{M}$ & $\mathrm{M}$ & $\mathrm{M}$
\end{tabular}

a

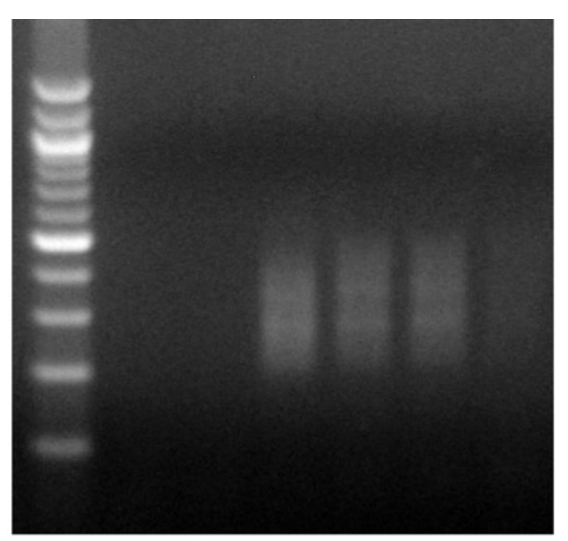

b

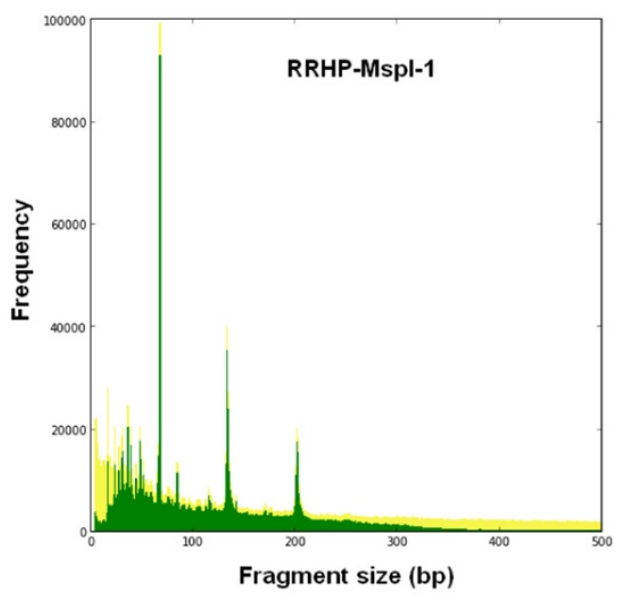

C

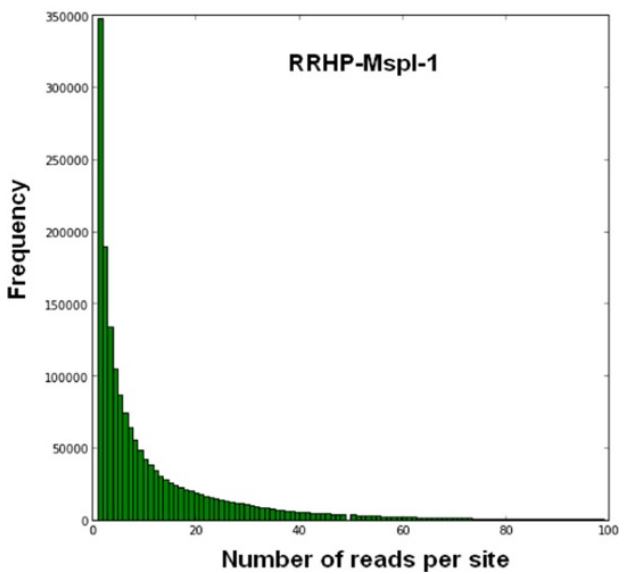

Figure $\mathbf{2}$ Library and sequencing characteristics of RRHP. (a) RRHP libraries prepared with different DNA input, with (+) or without (-) $\beta G T$, were digested by either Mspl (M) or Hpall (H). L is 100 bp Marker (Zymo Research). (b) Mspl digested fragments (green) detected by the RRHP assay overlaid with the fragments (yellow) generated from an in silico simulation. (c) The frequency distribution of $5 \mathrm{hmC}$ sites with different read coverage profiled by the RRHP assay. of RRHP, we also observed a substantial overlap of $5 \mathrm{hmC}$ positions only within the methylated sites detected by RRBS (Additional file 3).

\section{Annotative characteristics and unique features}

Functional annotation analysis of our RRHP data (Additional file 4) revealed a large number of $5 \mathrm{hmC}$ sites profiled were located within annotated genes, especially introns (45\%), 5'UTR (12\%), and 3'UTR (5\%). Interestingly, only $10 \%$ of the total $5 \mathrm{hmC}$ sites profiled by RRHP overlapped with CpG islands despite the intrinsic bias of the assay towards high CpG densities; nevertheless, $88 \%$ of the CpG islands of the entire genome were covered by at least one $5 \mathrm{hmC}$ site. Another $8 \%$ of $5 \mathrm{hmC}$ sites were mapped to promoters, predominately in high CpG promoters (HCP), and $82 \%$ of gene promoters were covered by at least one $5 \mathrm{hmC}$ site. We also observed overlap of $5 \mathrm{hmC}$ sites to diverse regulatory elements such as histone methylation, indicating broad survey of regions other than $\mathrm{CPG}$ islands and promoters. To examine whether $5 \mathrm{hmC}$ sites are associated with genes of specific functions, we performed gene ontology analysis using GREAT [11], which allows functional analysis of cis-regulatory regions such as enhancers. Interestingly, no significant gene enrichment of any cellular processes was found to be associated with $5 \mathrm{hmC}$. The RRHP assay can also detect the strand distribution of $5 \mathrm{hmC}$ because library construction is directionally unbiased. By counting the number of reads with the CCGG junction generated at the sense or antisense strand, we can determine their respective $5 \mathrm{hmC}$ abundance. Consistent with previous observations [9], a large number of $\mathrm{CpG}$ sites showed an asymmetric distribution of $5 \mathrm{hmC}$ (Figure 4A). About 50,000 sites were found to have read counts with at least a two-fold difference between the strands. Further analysis indicated there were no obvious preference of $5 \mathrm{hmC}$ to either the coding strand or different annotated regions such as promoter, intron, exon, and so on, indicating that the distribution of $5 \mathrm{hmC}$ are random between the stands (Figure 4B). In addition, since library preparation does not involve bisulfite conversion, we can directly identify single nucleotide polymorphisms (SNPs) within reads with high confidence (Additional file 5). Thus, both genetic variations and epigenetic modifications can be analyzed from a single data set.

\section{Cross-platform validations and correlation}

To further evaluate the reliability of the assay, we also performed genome-wide and locus-specific validations using a JBP-1-based enrichment assay [12] and a glucMSqPCR assay [13], respectively. Both methods showed strong correlations with de novo RRHP discoveries. RRHP allowed more sensitive detection of low-abundant $5 \mathrm{hmC}$ 
Table 1 Statistical analysis of sequencing reads from in silico simulation and RRHP experiment

\begin{tabular}{|c|c|c|c|c|c|c|c|}
\hline & $\begin{array}{l}\text { Simulation } \\
40-430 \mathrm{bp}\end{array}$ & $\begin{array}{l}\text { RRHP-Hpall } \\
(0.5 \mu \mathrm{g})\end{array}$ & $\begin{array}{l}\text { RRHP-Mspl-1 } \\
(0.5 \mu \mathrm{g})\end{array}$ & $\begin{array}{l}\text { RRHP-Mspl-2 } \\
(0.5 \mu \mathrm{g})\end{array}$ & $\begin{array}{l}\text { RRHP-Mspl-3 } \\
(0.1 \mu \mathrm{g})\end{array}$ & $\begin{array}{l}\text { RRHP } \\
\text { (- } \beta \text { gt control ) }\end{array}$ & RRBS \\
\hline Total reads & $1,845,334$ & $23,702,341$ & $23,383,403$ & $9,505,230$ & $19,539,657$ & 5,518 & $41,066,513$ \\
\hline Mapped reads & $1,845,025$ & $20,605,538$ & $22,271,499$ & $9,017,016$ & $18,482,119$ & 4,373 & $15,668,523$ \\
\hline Mappability (\%) & 99.98 & 86.93 & 95.24 & 94.86 & 94.59 & 79.25 & 38.15 \\
\hline Tagged reads (n) & $1,845,025$ & $17,763,501$ & $21,081,749$ & $8,531,903$ & $17,328,911$ & 3,230 & NA \\
\hline Tagged reads (\%) & 100 & 86 & 95 & 95 & 94 & 74 & NA \\
\hline 5 hmc sites (n) & $1,845,014$ & $1,878,394$ & $1,737,993$ & $1,550,791$ & $1,674,080$ & 3,171 & $5,330,488$ \\
\hline
\end{tabular}

sites, which cannot be resolved with enrichment approaches. We processed RRHP data with MACS program [14] to create a peak track analogous to that of enrichment sequencing. Overlay of the tracks revealed close similarity of peak distributions over $5 \mathrm{hmC}$-containing regions (Figure $5 \mathrm{~A}$ ). Of the 4,000 peaks identified by the JBP-1 based hMeDIP-Seq, 40\% overlapped with at least one $5 \mathrm{hmC}$ site profiled by RRHP. However, only $0.5 \%$ of the $5 \mathrm{hmC}$ sites detected by RRHP fell within the peak regions of hMeDIP-Seq. This indicates RRHP is more sensitive in identifying $5 \mathrm{hmC}$ sites although it is limited by the distribution of restriction sites. Importantly, RRHP peaks can be confidently called at regions that would have fallen at or below background with enrichment sequencing. For further validation using glucMS-qPCR, we selected two different loci not identified as a $5 \mathrm{hmC}$ peak in hMeDIP-Seq but were detected by RRHP with 114 and nine reads. Conveniently, the RRHP and glucMS-qPCR assays allowed for straightforward cross-validation due to the shared foundation of glucosyl-sensitive digestion reactivities. In the simplest embodiment of the method, each $5 \mathrm{hmC}$ positioned at a MspI junction was profiled by creating primers flanking the site. Genomic DNA was treated or mock-treated with $\beta$-GT, subjected to MspI digestion, and then amplified by qPCR to confirm glucosyl protection at the locus and to quantify the abundance of $5 \mathrm{hmC}$ at the position by $\Delta \mathrm{Ct}$. The $\beta-\mathrm{GT}$ treated samples had a lower $\mathrm{Ct}$ than the mock- $\beta-\mathrm{GT}$ treated, indicating the presence of $5 \mathrm{hmC}$ at the two loci (Figure 5B). The $5 \mathrm{hmC}$ abundance was calculated to be approximately $97.62 \%$ and $49.67 \%$, respectively, for these two sites using the formula mentioned in the method section.

\section{$5 \mathrm{hmC}$ profiling in breast and liver cancer samples by RRHP}

Previous studies on the role of $5 \mathrm{hmC}$ in cancer have shown loss of $5 \mathrm{hmC}$ is commonly associated with tumor development in both hematological diseases and solid tumors [15]. However, it is not clear whether the decrease of $5 \mathrm{hmC}$ is a result of global $5 \mathrm{mC}$ reduction, which is also a hallmark of tumorgenesis, or due to a different epigenetic regulation. Since those studies utilized either liquid chromatography-mass spectrometry (LC-MS/MS) or antibody-based immune dot blots and immunohistochemistry to measure $5 \mathrm{hmC}$ levels, $5 \mathrm{hmC}$ alternation with gene-level resolution cannot be detected. To gain a further understanding of how $5 \mathrm{hmC}$ loss is involved in tumor initiation or progression, it is necessary to identify the genes and signaling pathways that are regulated by changes in $5 \mathrm{hmC}$. To this regard, we performed a pilot study for two types of paired solid tumor samples (breast and liver tumors as well as their adjacent normal tissues) using RRHP. In both cases, tumor and normal samples showed a very similar genomic distribution in terms of the total number of $5 \mathrm{hmC}$ sites detected. However, the $5 \mathrm{hmC}$ abundance at each CpG site was altered globally when we did a side-by-side comparison between the paired samples, which is reflected by the read counts change (as shown in Additional file 6). This result is in good agreement with previous observations that $5 \mathrm{hmC}$ levels tend to decrease in tumor samples $[16,17]$. GREAT analyses were also performed using the top 2,000 significantly different $5 \mathrm{hmC}$ sites between the paired tumor samples (Additional file 7). Genes enriched from breast tumor analysis were found to regulate processes such as osteoblast differentiation, gliogenesis, actin filament bundle assembly, and so on, which all have been proved to be associated with breast cancer metastasis previously [18-20]. On the other hand, genes enriched from the liver tumor analysis were more related to metabolic or biosynthetic regulation of sterol, steroid, ketone, lipid, fatty acid, and so on. The majority of genes with $5 \mathrm{hmC}$ reduction had been shown to be downregulated in hepatocellular carcinoma, indicating $5 \mathrm{hmc}$ loss might be used to transcriptionally inactivate certain tumor suppressors since early studies had shown that $5 \mathrm{hmC}$, especially those located in gene bodies, were associated with transcriptional activity. As an example, we checked the $5 \mathrm{hmC}$ levels of two tumor suppressors: LZTS1 in breast cancer and XPO4 in liver cancer [21,22]. By examining 75 primary breast cancers and 12 normal breast tissues, Wielscher et al. had recently found that LZTS1 had significantly lower $5 \mathrm{hmC}$ content in tumors compared to 

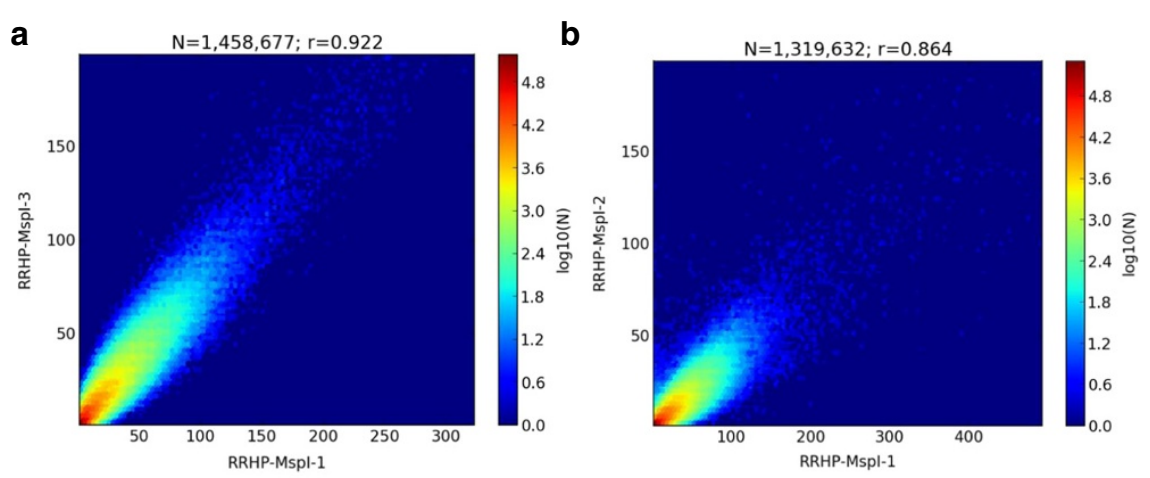

C

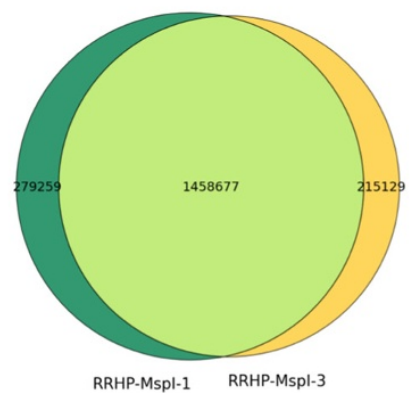

e
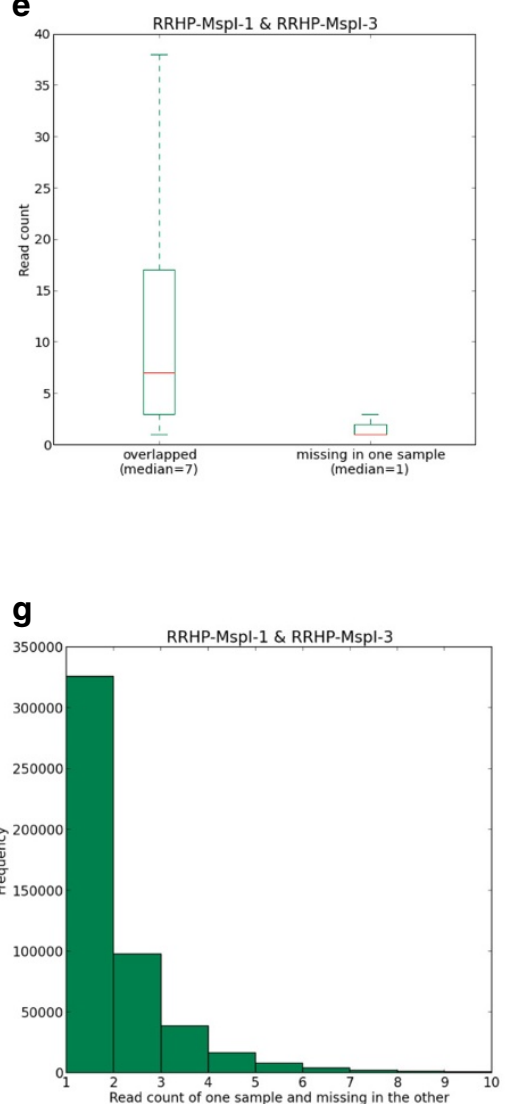

d

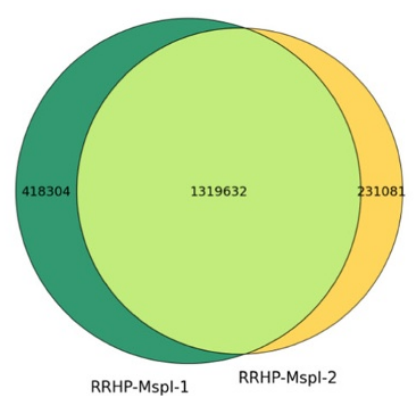

f

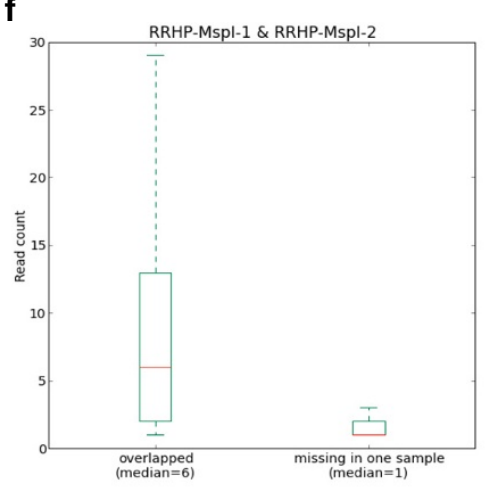

h

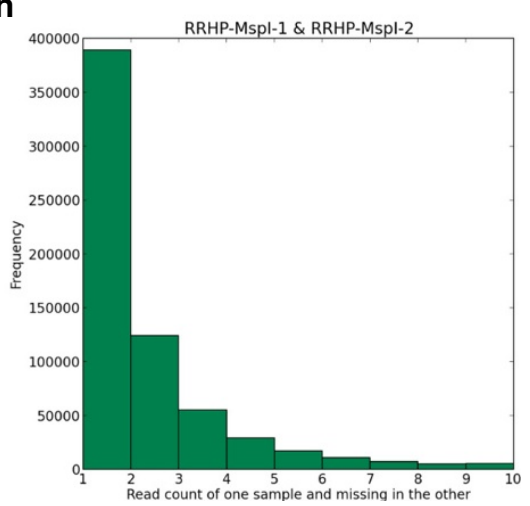

Figure 3 (See legend on next page.) 
(See figure on previous page.)

Figure 3 Analysis of RRHP libraries prepared with different DNA input and sequencing depth. (a) Scatter plot of the read counts between RRHP-Mspl-1 and RRHP-Mspl-3. (b) Scatter plot of the read counts between RRHP-Mspl-1 and RRHP-Mspl-2. (c) Venn diagrams show RRHP-Mspl-1 and RRHP-Mspl-3 have 1.45 million 5hmC sites in common while there are 279,259 unique sites in RRHP-Mspl-1 and 215,129 unique sites in RRHP-Mspl-3. (d) Venn diagrams show RRHP-Mspl-1 and RRHP-Mspl-2 have 1.3 million sites in common while there are 418,304 unique sites in RRHP-Mspl-1 and 231,081 unique sites in RRHP-Mspl-3. (e) Box plots shows that 5hmC sites overlapping in RRHP-Mspl-1 and RRHP-Mspl-3 have higher read counts on average than non-overlapping sites. (f) Box plot shows 5hmC sites overlapping in RRHP-Mspl-1 and RRHP-Mspl-2 have higher read counts on average than non-overlapping sites. (g) Distribution of read counts of non-overlapping 5hmC sites between RRHP-Mspl-1 and RRHP-Mspl-3. (h) Distribution of read counts of non-overlapping 5hmC sites between RRHP-Mspl-1 and RRHP-Mspl-2.

normal breast tissues in the region between the 5'UTR to the second exon while no significant differences were observed for $5 \mathrm{mC}$. Correspondingly, the LZTS1 mRNA expression was reduced in the tumor samples, suggesting a strong influence of $5 \mathrm{hmC}$ on mRNA expression. Consistent with these results, the genome browser track from RRHP for the same region again showed a decrease of $5 \mathrm{hmC}$ in tumor in comparison to its adjacent normal (Figure 6a). This pattern was also observed for the liver tumor suppressor XPO4 (Figure 6b).

\section{Discussion}

RRHP features a rapid workflow, avoids harsh chemical modification, and allows processing of DNA inputs as low as $100 \mathrm{ng}$. Also, RRHP is a positive display that eliminates the need for parallel subtractive sequencing as required by oxBS-Seq and does not need high sequencing depth to detect low $5 \mathrm{hmC}$ abundances as required by TAB-Seq, which needs an average of $26.5 \times$ read coverage to resolve a single $5 \mathrm{hmC}$ site with $20 \%$ abundance at a false discovery rate (FDR) $<5 \%$. Under the same FDR, we found that RRHP was able to confidently detect approximately one million $5 \mathrm{hmC}$ sites in human brain tissue with only 20 to 30 million reads. Since brain tissues have the highest $5 \mathrm{hmC}$ content compared to other tissues examined thus far, such sequencing depth should be sufficient for other tissues. However, it would still be helpful if a pilot library for an unknown sample was sequenced with a higher depth and then analyzed as a function of the total number of reads by down-sampling the data to determine the minimal sequencing depth required. This will enable the user to adjust the sequencing depth accordingly and make sequencing runs more cost-efficient. It is also worth noting that samples which need comparative analysis should be sequenced with the same depth or normalization is required. Typically, data for the same sample from runs with different sequencing depth can be normalized by the total number of reads. However, due to the positive display nature of RRHP, the total number of sequencing reads is not only associated with sequencing depth but also related to $5 \mathrm{hmC}$ abundance. Therefore, normalization by the total number of reads is inappropriate when comparing different samples since the number of sequencing reads is an indication of $5 \mathrm{hmC}$ abundance. Thus, $5 \mathrm{hmC}$ sites with housekeeping characteristics are needed to serve as an internal control to normalize samples. Alternatively, a spikein control with various $5 \mathrm{hmC}$ levels would be helpful for normalization. Currently, we maintain the relative $5 \mathrm{hmC}$ abundance between samples by multiplexing samples with equal volume, rather than equal mass, for libraries prepared in parallel (that is, same DNA input, same purification, same amplification, and so on). For example, we multiplexed sample RRHP-MspI-2 with half the volume that of RRHP-MspI-1, and as expected, the total number of reads for RRHP-MspI-2 was half of RRHP-MspI-1 (Table 1). Without data normalization, the correlation

Table 2 Calculated false calling rates of RRHP for various read counts

\begin{tabular}{|c|c|c|c|c|c|c|}
\hline \multirow[t]{2}{*}{ Read cutoff no. } & \multicolumn{2}{|c|}{ RRHP-Mspl-1 } & \multicolumn{2}{|c|}{ RRHP-Mspl-2 } & \multicolumn{2}{|c|}{ RRHP-Mspl-3 } \\
\hline & Error rate & Error count & Error rate & Error count & Error rate & Error count \\
\hline 1 & 0.358 & 585 & 0.474 & 775 & 0.242 & 396 \\
\hline 2 & 0.143 & 233 & 0.191 & 312 & 0.106 & 173 \\
\hline 3 & 0.057 & 93 & 0.079 & 129 & 0.054 & 88 \\
\hline $4^{a}$ & 0.026 & 42 & 0.028 & 46 & 0.029 & 48 \\
\hline 5 & 0.02 & 32 & 0.013 & 21 & 0.02 & 33 \\
\hline 6 & 0.015 & 24 & 0.007 & 11 & 0.015 & 25 \\
\hline 7 & 0.009 & 15 & 0.004 & 7 & 0.011 & 18 \\
\hline 8 & 0.008 & 13 & 0.004 & 7 & 0.008 & 13 \\
\hline 9 & 0.006 & 9 & 0.004 & 6 & 0.006 & 10 \\
\hline 10 & 0.005 & 8 & 0.003 & 5 & 0.004 & 7 \\
\hline
\end{tabular}

${ }^{a}$ Minimal read counts required for an error rate $<0.05$. 

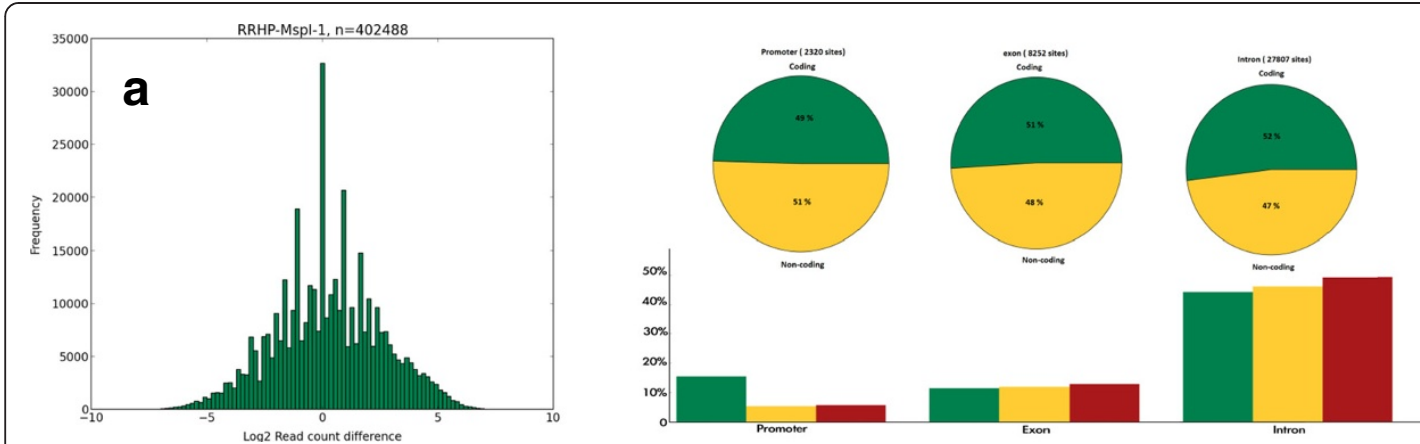

b

Figure $45 \mathrm{hmC}$ is asymmetrically distributed between the forward and reverse strand throughout the genome. (a) Distribution of the read count difference (log2 scale) between the forward and reverse strand for sample RRHP-Mspl-1. (b) CpG sites with asymmetric 5hmC distribution between the forward and reverse strand were analyzed according to gene annotation (column plot) and coding (pie chart). Legend for annotation column plot - Green: detectable Mspl sites; Yellow: Mspl site with 5hmC detected from both strands; Red: Mspl sites with two-fold $5 \mathrm{hmC}$ difference between the strands. Legend for pie chart - Green: coding strand; Yellow: non-coding strand.

between the two samples was 0.864 , indicating little variation in the sample preparation (Figure $3 \mathrm{~b}$ ). Also, from our comparison of the paired tumor samples, our results were in agreement with previously published data that showed tumors have less $5 \mathrm{hmC}$ abundance.

The high sensitivity and low background associated with RRHP allows for both qualitative and relative quantitative descriptions of $5 \mathrm{hmC}$ at genomic loci, resulting in high correlation with previously described interrogative methods such as hMeDIP-Seq and glucMS-qPCR. The method detects $5 \mathrm{hmC}$ in a strand-specific fashion and can couple analyses of epigenetic modifications with genomic variation, such as SNP detection. When combined with RRBS data, the method allows for high resolution and direct correlation of $5 \mathrm{mC}$ and $5 \mathrm{hmC}$ positions. This system can be adapted for any platform (such as ion torrent PGM and so on) that utilizes adapterized libraries, and by using other glucose-sensitive restriction enzymes for fragmentation and library digestion, we can profile $5 \mathrm{hmC}$ sites in alternative CpG motifs as well as non-CpG contexts. This principle can also be applied in mapping other epigenetic modifications, such as $5 \mathrm{fC}, 5 \mathrm{CaC}$ and N6-methyladenine $(6 \mathrm{~mA})$ by using alternative restriction enzymes. In addition, three other enzyme-based methods were also recently developed for genome-wide $5 \mathrm{hmC}$ profiling including Aba-Seq, HELP-GT assay and HMST-Seq [23-25]. Aba-Seq utilizes a DNA-modification dependent restriction endonuclease, AbaSI, coupled with sequencing. AbaSI recognizes glucosylated $5 \mathrm{hmC}$ with high specificity and generates a double strand break 11-13 bp downstream of the recognition site. However, this enzyme prefers sites with two cytosines positioned symmetrically around the cleavage site, and the cleavage efficiency is lower when only one of the two cytosines is a glucosyl-5hmC. Putative $5 \mathrm{hmC}$ sites are indirectly deduced by checking for the presence of a cytosine at the expected distances from either side of the mapped cleavage sites. There are two major limits for this method: first, certain $5 \mathrm{hmC}$ sites may not be detected due to the low cleavage efficiency caused by the absence of symmetric pattern of the recognition site. Second, it causes assignment ambiguity to the exact cytosine in categories which has 2CGs or $2 \mathrm{CHs}$ at the symmetric recognition site, and these sites account for $13 \%$ of all identified cleavage sites, according to the authors. In addition, Aba-Seq requires a much higher sequencing depth; over 200 million reads is needed for an Aba-Seq library, making it not cost competitive to RRHP. The other two assays, HELP-GT and HMST-Seq, are more similar to RRHP in terms of the restriction enzyme used and the genomic coverage, but both of them are negative display methods and require subtractive sequencing. In other words, two libraries for each sample have to be sequenced in order to infer the $5 \mathrm{hmC}$ status for a $\mathrm{CpG}$ site, and it is challenging to normalize the data for subtraction given a variety of factors which may affect the read counts and distribution between the two libraries. Lastly, both HELP-GT and HMST-Seq assays have a very complicated workflow which requires multiple enzymatic digestions, sequential adapterization, bead capture or in vitro transcription, making it not ideal for samples with low DNA input.

\section{Conclusions}

Here we present a novel approach, RRHP, for genome-wide profiling of $5 \mathrm{hmC}$, which exploits $\beta$-glucosyltransferase $(\beta-G T)$ to inhibit restriction digestion at adapters ligated to a genomic library, such that only fragments presenting glucosylated $5 \mathrm{hmC}$ residues at adapter junctions will be amplified and sequenced. This assay profiles $5 \mathrm{hmC}$ sites with single-base resolution in a strand-specific fashion. When combined with existing RRBS data, it allows for simultaneous comparison of $5 \mathrm{mC}$ and $5 \mathrm{hmC}$ at a specific site. We find that this assay is a robust and cost-efficient tool for profiling $5 \mathrm{hmC}$ across the genome. 


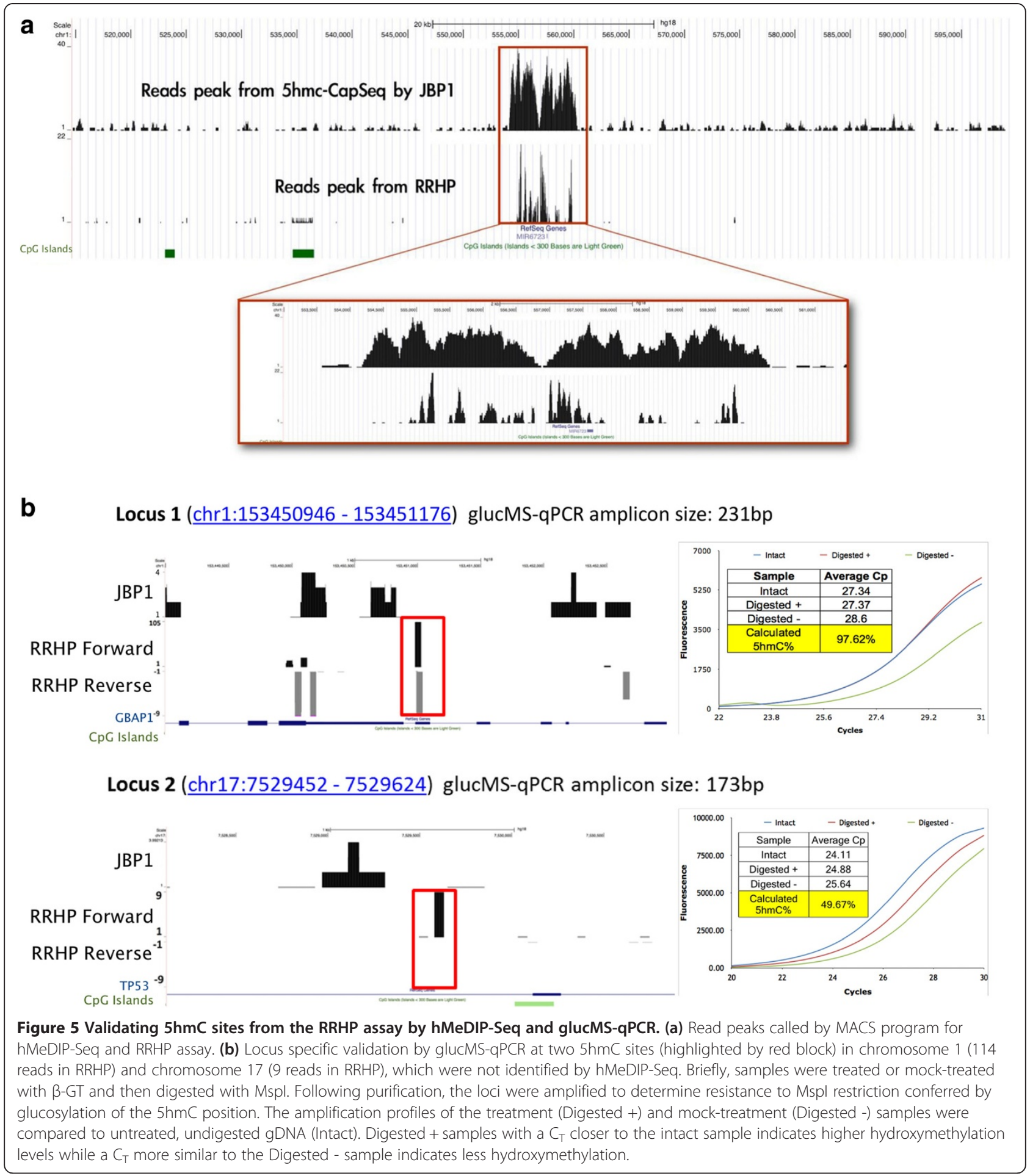

\section{Methods}

\section{Adapter design and construction}

P5CCG and P7CG adapter pairs were constructed in a manner that allowed for 5 '-CG overhangs instead of the standard 5 ' $-\mathrm{T}$ overhangs of the Illumina TruSeq P5 and P7 adapters. In the P5CCG adapter pair, CCGG is retained at the junction following ligation to a library fragment while in the P7CG adapter pair, the CCGG junction becomes TCGG at ligation and is no longer sensitive to HpaII or MspI restriction. For both adapter pairs, two long oligos were hybridized with their respective complementary short oligos at $50 \mu \mathrm{M}$ with a 


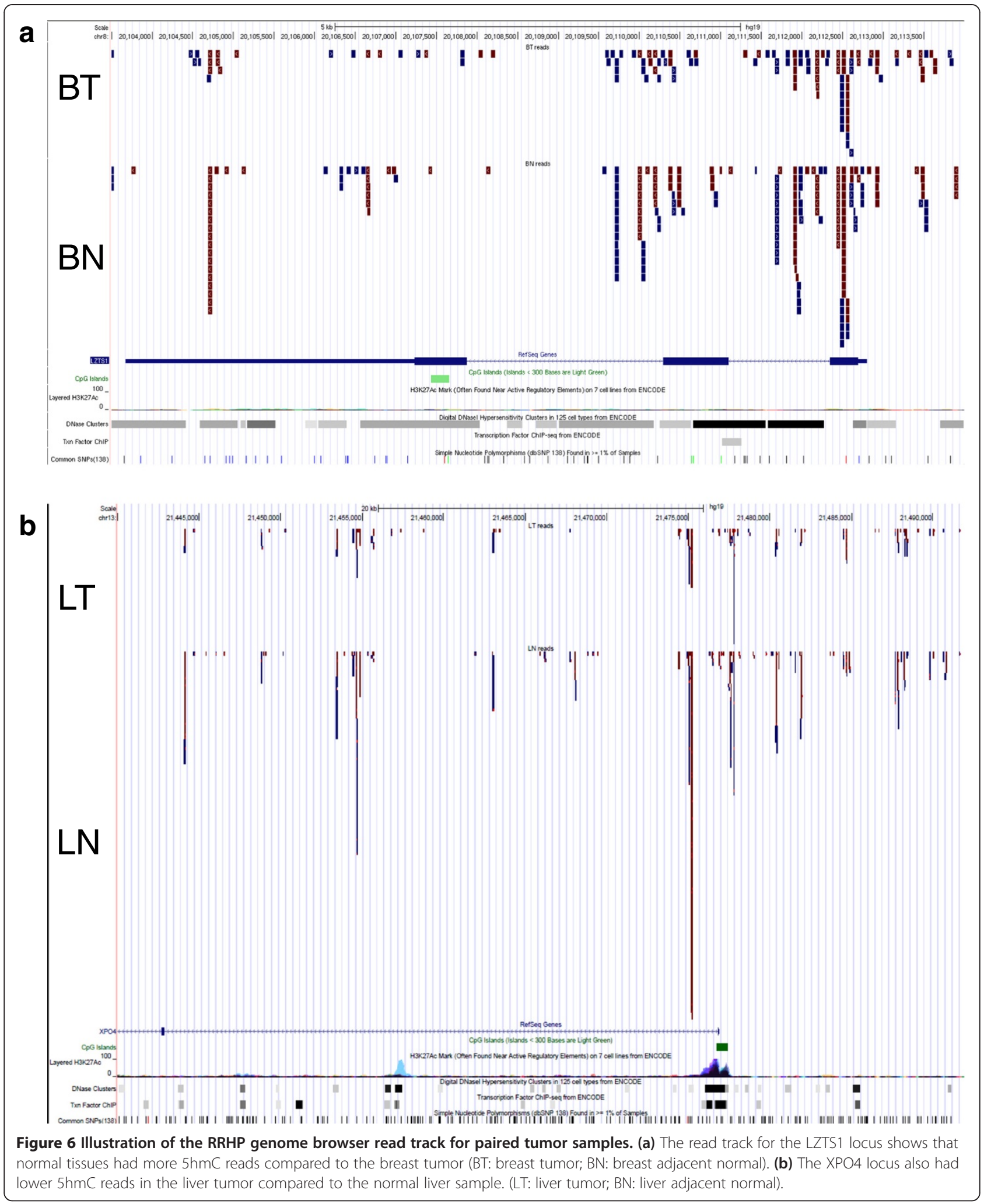

slow ramp-down $\left(0.1^{\circ} \mathrm{C} / \mathrm{s}\right)$ from $95^{\circ} \mathrm{C}$ to $12^{\circ} \mathrm{C}$ in oligo hybridization buffer $(50 \mathrm{mM} \mathrm{NaCl}, 1 \mathrm{mM}$ Tris- $\mathrm{HCl} \mathrm{pH} \mathrm{8.0,}$ $100 \mu \mathrm{M}$ EDTA). The P5 adapter pair was prepared from
HPLC-purified oligos (IDT): 5'-ACACTCTTTCCCTACA CGACGCTCTTCCGATCTC-3' (long) and 5'-CGGAGA TCGGAAGAG-3ddC -3' (short). The P7 adapter pair 
was prepared from the oligos: 5'-GTGACTGGAGTTC AGACGTGTGCTCTTCCGATCT-3' (long) and 5' - CGA GATCGGAAGAG-3ddC -3' (short).

\section{RRHP library construction and sequencing}

Genomic DNA from human male cerebellum or tumor and adjacent normal tissue was purified via phenol: chloroform extraction and digested for $8 \mathrm{~h}$ at $37^{\circ} \mathrm{C}$ with $20 \mathrm{U}$ MspI (NEB). Following digestion, the enzyme was inactivated at $65^{\circ} \mathrm{C}$ for $15 \mathrm{~min}$ and fragmented gDNA was purified using the DNA Clean and Concentrator kit (Zymo Research). For adapterization, 100 or $500 \mathrm{ng}$ of fragmented DNA was ligated overnight at $16^{\circ} \mathrm{C}$ with 400 U T4 DNA Ligase (NEB) and $500 \mathrm{nM}$ modified P5 and P7 adapters (IDT), such that the CCGG junction was retained at the P5 adapter and destroyed in the P7 adapter (see 'Adapter construction' for sequence detail). After overnight incubation, adapters were extended with $2 \mathrm{U}$ GoTaq (Promega) and $500 \mu \mathrm{M}$ dNTPs (Zymo Research) at $72^{\circ} \mathrm{C}$ for $30 \mathrm{~min}$. Following extension, adapterized libraries were purified with the DNA Clean and Concentrator kit. Adapterized libraries were glucosylated with $10 \mathrm{U} \beta$-GT (Zymo Research) and $100 \mathrm{nM}$ UDPG at $37^{\circ} \mathrm{C}$ for $4 \mathrm{~h}$. For negative control reactions, $\beta$-GT was omitted from the incubation. To all of the reactions, $20 \mathrm{U}$ of MspI or HpaII were then added and incubated at $37^{\circ} \mathrm{C}$ overnight. After digestion, an additional $20 \mathrm{U}$ of MspI or HpaII were added and allowed to incubate for an additional $1 \mathrm{~h}$. Enzymes were heat inactivated at $65^{\circ} \mathrm{C}$ and libraries were purified with the DNA Clean and Concentrator kit. Purified libraries were then loaded in a 2.5\% (w/v) 50:50 NuSieve:agarose gel and electrophoresed. Size-selected libraries were cut from 110 to $500 \mathrm{bp}$ and purified with the Zymoclean Gel DNA Recovery Kit (Zymo Research). Finalized libraries were then amplified with $500 \mathrm{nM}$ P5/P7 barcoding primers (IDT) in OneTaq 2X Master Mix (NEB) with the thermal profile: $94^{\circ} \mathrm{C}$ for $30 \mathrm{~s}, 58^{\circ} \mathrm{C}$ for $30 \mathrm{~s}$, and $68^{\circ} \mathrm{C}$ for $30 \mathrm{~s}$, repeated for 10 cycles. Amplifications were sampled for visualization on a $2 \%$ agarose and purified with the DNA Clean and Concentrator kit. For sequencing, equal volumes of each amplified library were pooled and diluted to $8 \mathrm{pM}$ for $50 \mathrm{bp}$ singleton reads on the Illumina HiSeq 2000 (Illumina).

\section{Bioinformatic processing and statistical analyses}

Sequencing reads from the RRHP assay were first processed to trim off low quality bases and the P7CG adapter at the $3^{\prime}$ end of the reads and then aligned to the hg18 build of the human genome using Bowtie0.12.8 and its default parameters with -best. Aligned reads with CCGG tag at $5^{\prime}$ end were counted. The correlation analysis between the different RRHP libraries were performed by comparing the presence of the tagged reads at each profiled MspI site, and the Pearson's coefficient was calculated accordingly. The reads for RRBS library were processed as previously reported. Gene ontology analysis was performed using the Genomic Regions Enrichment of Annotations Tool (GREAT) [11].

\section{JBP-1-mediated enrichment sequencing library preparation and analysis}

The enrichment sequencing libraries were prepared from 1 ug gDNA fragmented with dsDNA Shearase (Zymo Research). Fragments were then A-tailed with Klenow exo- fragment (NEB) and ligated to adapters per standard Illumina library preparation protocols. Libraries were glucosylated with $\beta$-GT and enriched via incubation with immobilized JBP-1 using the Quest $5 \mathrm{hmC}$ DNA Enrichment Kit (Zymo Research) per the manufacturer's protocol. Libraries were subjected to limited amplification, purified, and sequenced on the Genome Analyzer IIX platform (Illumina). Resulting reads were trimmed for adapters, aligned to hg18 with Bowtie, and analyzed for enrichment peak calling in MACS.

\section{glucMS-qPCR validation of de novo $5 \mathrm{hmC}$ discovery loci}

A total of $100 \mathrm{ng}$ of gDNA from the same human male cerebellum sample was glucosylated with $10 \mathrm{U} \beta$-GT (Zymo Research) and $100 \mathrm{nM}$ UDPG (Zymo Research) or mock-treated without enzyme at $37^{\circ} \mathrm{C}$ for $2 \mathrm{~h}$. To the same reactions, $20 \mathrm{U}$ of MspI (NEB) were added and incubated for an additional $2 \mathrm{~h}$. Following heat inactivation at $65^{\circ} \mathrm{C}$ for $15 \mathrm{~min}$, reactions were purified with the DNA Clean and Concentrator kit (Zymo Research) and quantified. $10 \mathrm{ng}$ of each treatment group was utilized for qPCR in triplicate with QuestTaq qPCR Master Mix (Zymo Research) and $200 \mathrm{nM}$ primers (IDT). Reactions were amplified on a CFX96 cycler (Bio-Rad) with the thermal profile: $95^{\circ} \mathrm{C}$ for $3 \mathrm{~min}, 40$ cycles of $95^{\circ} \mathrm{C}$ for $30 \mathrm{~s}, 60^{\circ} \mathrm{C}$ for $20 \mathrm{~s}, 72^{\circ} \mathrm{C}$ for $20 \mathrm{~s}$, and then a final extension at $72^{\circ} \mathrm{C}$ for $1 \mathrm{~min}$ before a $4^{\circ} \mathrm{C}$ hold. All amplifications were then subjected to melt curve analysis to ensure specific amplification and identity. $C p$ values were averaged from three technical replicates for each treatment and $5 \mathrm{hmC} \%$ was calculated using the equation ((Digested -)-(Digested +$) /($ Digested -) $($ Intact $)) \times 100 \%$.

Primers for glucMS-qPCR Validation:

Locus 1 Chr1 : 153450946 - 153451176

Fwd: 5' CTTCAGCCCACTTCCCAGAC

Rev: 5' GTGGGTGGGCGACTTCTTAG

Locus 2 Chr17: 7529452 - 7529624

Fwd: 5' AAGGACAGAAGCCCGACAAA

Rev: 5' CAGCTATTCGGGAGGGTGAG 


\section{Data access}

The RRHP sequencing data from this study have been submitted to NCBI's Gene Express Omnibus (GEO) under accession number GSE49546.

\section{Additional files}

Additional file 1: The frequency distribution of the $5 \mathrm{hmC}$ sites with different read coverage profiled by RRHP assay.

Additional file 2: Pairwise comparison between libraries prepared with different inputs. (a) Venn diagrams show number of $5 \mathrm{hmC}$ sites in RRHP-Mspl-1 and RRHP-Mspl-3 with 725,464 sites in common and 154,226 unique sites in RRHP-Mspl-1 and 99,237 unique sites under the condition of five reads cutoff. (b) Plotting Pearson's correlation coefficient from the comparison between RRHP-Mspl-1 and RRHP-Mspl-3 against sequencing read cutoff.

Additional file 3: Integrative representation of RRBS and RRHP data for RASSP1 gene in UCSC genome browser. CPG sites with low methylation in RRBS show no or few reads in RRHP. Most of the RRHP reads were found in the gene promoter of RASSP1, which also overlap with H3K4me1 and H3K4me3 modification as well as the DNasel hypersensitive cluster in that region (highlighted by green frame). Interestingly the reverse strand (coding stand) has higher hydroxymethylation than the forward strand (non-coding strand) as indicated by the number of the reads in each strand (arrows in the reads indicate either forward or reverse strand). Red: non methylation, yellow: methylation (The number next to each CpG site indicates number of methylated reads in total reads; for example, 4/34 means four methylated read in total 34 reads covering that $C p G$ site)

Additional file 4: Breakdown of $5 \mathrm{hmC}$ sites profiled by RRHP into specific annotated genomic elements and binding locations. All annotations were obtained from UCSC Genome Browser (hg18). CpG islands were directly obtained from CpG Islands track. 5'UTR, 3'UTR, promoter, exons, and introns were based on RefSeq Genes track. Promoter was defined as $1 \mathrm{~kb}$ upstream and downstream of TSS. HighCpG promoters (HCPs), weak CpG islands or intermediate- $\mathrm{CpG}$ promoters (ICPs), and sequences with no local enrichment of CpGs or low-CpG promoters (LCPS) were calculated based on Weber et al. [26]. Coordinates for regions of $7 \times$ regulatory potential or regions (average score $>0.5$ ) conserved in human, chimpanzee, macaque, mouse, rat, dog, and cow were obtained from King et al. [27]. Histone ChIP-chip binding data was H1ES H3K4me3 and H1ES H3K27me3 tables from Broad Histone track. Bivalent regions were areas that overlapped with both H3K4me3 and H3K27me3 peaks. RRHP regions overlapping with H3K4me3 peaks only were called $\mathrm{H} 3 \mathrm{~K} 4 \mathrm{me}$; regions overlapping with $\mathrm{H} 3 \mathrm{~K} 27$ me3 peaks only were called H3K27me3.

Additional file 5: Illustration of RRHP data in UCSC genome browser. The strandedness of each CpG site in the RRHP-CpG track is indicated by blue (forward strand) and red (reverse strand), and the number next to each CpG indicates the read coverage. The same color code applies in the RRHP read track with a letter indicating the SNPs on each strand.

Additional file 6: Box plot shows read count distribution for all the common sites between the paired tumors.

Additional file 7: GREAT analysis for the top $2,0005 \mathrm{hmC}$ sites between paired tumor and normal sample. The input of GREAT is the combination of top 1,000 sites from intersection data (all sites must be non-zero) and top 1,000 sites form symmetric difference sites (one sites must be zero). No site selection and normalization were applied to the above data set. The ranking criteria are absolute values of log ratio (tumor count/normal count) from large to small.

\section{Authors' contributions}

XS conceived and designed the study. AP and DT constructed libraries and performed sequencing experiments. THC performed bioinformatic processing of sequencing data and all subsequent statistical analyses. AP, XJ, and XS wrote the paper. All authors read and approved the final manuscript.

\section{Acknowledgements}

We thank M. Van-Eden, K. Booher, J. Claypool, and P. Shi for helpful comments on the manuscript.

\section{Author details}

'Zymo Research Corporation, 17062 Murphy Ave., Irvine, CA 92614, USA.

${ }^{2}$ Current address: 5200 Illumina Way, La Jolla 92122, USA.

Received: 1 April 2014 Accepted: 28 August 2014

Published online: 24 September 2014

\section{References}

1. Kriaucionis S, Heintz N: The nuclear DNA base 5-hydroxymethylcytosine is present in purkinje neurons and the brain. Science 2009, 324:929-930.

2. Tahiliani M, Koh KP, Shen Y, Pastor WA, Bandukwala H, Brudno Y, Agarwal S, Lyer LM, Liu DR, Aravind L, Rao A: Conversion of 5-methylcytosine to 5-hydroxymethylcytosine in mammalian DNA by MLL partner TET1. Science 2009, 324:930-935.

3. Guo JU, Su Y, Zhong C, Ming GL, Song H: Hydroxylation of 5-methylcytosine by TET1 promotes active DNA demethylation in the adult brain. Cell 2011, 145:423-434.

4. He YF, Li BZ, Li Z, Liu P, Wang Y, Tang Q, Ding J, Jia Y, Chen Z, Li L, Sun Y, Li X, Dai Q, Song CX, Zhang K, He C, Xu GL: Tet-mediated formation of 5-carboxylcytosine and its excision by TDG in mammalian DNA. Science 2011, 333:1303-1307.

5. Globisch D, Münzel M, Müller M, Michalakis S, Wagner M, Koch S, Brückl T, Biel M, Carell T: Tissue distribution of 5-hydroxymethylcytosine and search for active demethylation intermediates. PLOS ONE 2010, 5:e15367.

6. Jin SG, Jiang Y, Qiu R, Rauch TA, Wang Y, Schackert G, Krex D, Lu Q, Pfeifer GP: 5-Hydroxymethylcytosine is strongly depleted in human cancers but its levels do not correlate with IDH1 mutations. Cancer Res 2011, 71:7360-7365.

7. Fu Y, He C: Nucleic acid modifications with epigenetic significance. Curr Opin Chem Biol 2012, 16:516-524

8. Booth MJ, Branco MR, Ficz G, Oxley D, Krueger F, Reik W, Balasubramanian S: Quantitative sequencing of 5-methylcytosine and 5-hydroxymethylcytosine at single-base resolution. Science 2012, 336:934-937.

9. Yu M, Hon GC, Szulwach KE, Song C, Zhang L, Kim A, Li X, Dai Q, Shen Y, Park B, Min J, Jin P, Ren B, He C: Base-resolution analysis of 5-hydroxymethylcytosine in the mammalian genome. Cell 2013, 149:1368-1380

10. Gu H, Smith ZD, Bock C, Boyle P, Gnirke A, Meissner A: Preparation of reduced representation bisulfite sequencing libraries for genome-scale DNA methylation profiling. Nat Protoc 2011, 6:468-481.

11. McLean CY, Bristor D, Hiller M, Clarke SL, Schaar BT, Lowe CB, Wenger AM, Bejerano G: GREAT improves functional interpretation of cis-regulatory regions. Nat Biotechnol 2010, 28:495-501.

12. Robertson AB, Dahl JA, Ougland R, Klungland A: Pull-down of 5-hydroxymethylcytosine DNA using JBP1-coated magnetic beads. Nat Protoc 2012, 7:340-350.

13. Doege CA, Inoue K, Yamashita T, Rhee DB, Travis S, Fujita R, Guarnieri $P$, Bhagat G, Vanti WB, Shih A, Levine RL, Nik S, Chen El, Abeliovich A: Early-stage epigenetic modification during somatic cell reprogramming by Parp1 and Tet2. Nature 2012, 488:652-655.

14. Zhang Y, Liu T, Meyer CA, Eeckhoute J, Johnson DS, Bernstein BE, Nusbaum C, Myers RM, Brown M, Li W, Liu XS: Model-based analysis of ChIP-Seq (MACS). Genome Biol 2008, 9:R137.

15. Mariani CJ, Madzo J, Moen EL, Yesilkanal A, Godley LA: Alterations of 5-hydroxymethylcytosine in human cancers. Cancers (Basel) 2013, 25:786-814.

16. Yang H, Liu Y, Bai F, Zhang JY, Ma SH, Liu J, Xu ZD, Zhu HG, Ling ZQ, Ye D, Guan $\mathrm{KL}$, Xiong $Y$ : Tumor development is associated with decrease of TET gene expression and 5-methylcytosine hydroxylation. Oncogene 2013, 32:663-669. 
17. Chen ML, Shen F, Huang W, Qi JH, Wang Y, Feng YQ, Liu SM, Yuan BF: Quantification of 5-methylcytosine and 5-hydroxymethylcytosine in genomic DNA from hepatocellular carcinoma tissues by capillary hydrophilic-Interaction liquid chromatography/ quadrupole TOF mass spectrometry. Clin Chem 2013, 59:824-832.

18. Mendoza-Villanueva D, Zeef $L$, Shore P: Metastatic breast cancer cells inhibit osteoblast differentiation through the Runx2/CBF $\beta$-dependent expression of the Wnt antagonist, sclerostin. Breast Cancer Res 2011, 13:R106.

19. Golshan M, Kuten A, William J, Richardson A, Modarressi A, Matulonis U: Metaplastic carcinoma of the breast with neuroglial differentiation. Breast 2006, 15:545-549.

20. Feng S, Cai M, Liu P, Wei L, Wang J, Qi J, Deng L: Atp6v1c1 may regulate filament actin arrangement in breast cancer cells. PLoS One 2014, 9:84833.

21. Wielscher M, Liou W, Pulverer W, Singer CF, Rappaport-Fuerhauser C, Kandioler D, Egger G, Weinhäusel A: Cytosine 5-hydroxymethylation of the LZTS1 gene is reduced in breast cancer. Trans/ Oncol 2013, 6:715-721.

22. Zender L, Xue W, Zuber J, Semighini CP, Krasnitz A, Ma B, Zender P, Kubicka S, Luk JM, Schirmacher P, McCombie WR, Wigler M, Hicks J, Hannon GJ, Powers S, Lowe SW: An oncogenomics-based in vivo RNAi screen identifies tumor suppressors in liver cancer. Cell 2008, 135:852-864.

23. Sun Z, Terragni J, Borgaro JG, Liu Y, Yu L, Guan S, Wang H, Sun D, Cheng X, Zhu Z, Pradhan S, Zheng Y: High-resolution enzymatic mapping of genomic 5-hydroxymethylcytosine in mouse embryonic stem cells. Cell Rep 2013, 3:567-576.

24. Gao F, Xia Y, Wang J, Luo H, Gao Z, Han X, Zhang J, Huang X, Yao Y, Lu H, Yi N, Zhou B, Lin Z, Wen B, Zhang X, Yang H, Wang J: Integrated detection of both 5-mC and 5-hmC by high-throughput tag sequencing technology highlights methylation reprogramming of bivalent genes during cellular differentiation. Epigenetics 2013, 8:421-429.

25. Bhattacharyya S, Yu Y, Suzuki M, Campbell N, Mazdo J, Vasanthakumar A, Bhagat TD, Nischal S, Christopeit M, Parekh S, Steidl U, Godley L, Maitra A, Greally JM, Verma A: Genome-wide hydroxymethylation tested using the HELP-GT assay shows redistribution in cancer. Nucleic Acids Res 2013, 41:e157.

26. Weber M, Hellmann I, Stadler MB, Ramos L, Pääbo S, Rebhan M, Schübeler D: Distribution, silencing potential and evolutionary impact of promoter DNA methylation in the human genome. Nat Genet 2007, 39:457-466.

27. King DC, Taylor J, Elnitski L, Chiaromonte F, Miller W, Hardison RC: Evaluation of regulatory potential and conservation scores for detecting cis-regulatory modules in aligned mammalian genome sequences. Genome Res 2005, 15:1051-1060.

\section{doi:10.1186/s13059-014-0456-5}

Cite this article as: Petterson et al:: RRHP: a tag-based approach for 5-hydroxymethylcytosine mapping at single-site resolution. Genome Biology 2014 15:456.

\section{Submit your next manuscript to BioMed Central and take full advantage of:}

- Convenient online submission

- Thorough peer review

- No space constraints or color figure charges

- Immediate publication on acceptance

- Inclusion in PubMed, CAS, Scopus and Google Scholar

- Research which is freely available for redistribution 